\title{
SCANNING ELECTRON MICROSCOPY AND ATOMIC FORCE MICROSCOPY OF CHITOSAN COMPOSITE FILMS
}

\author{
GALO CÁRDENAS ${ }^{a^{*}}$, PAOLA ANAYA ${ }^{a}$, RODRIGO DEL RIO ${ }^{b}$, RICARDO SCHREBLER *b, \\ CARLOS von PLESSING ${ }^{c}$, MARK SCHNEIDER ${ }^{d}$
}

\begin{abstract}
${ }^{a}$ Departamento de Polimeros, Facultad de Ciencias Químicas, Universidad de Concepción. Concepción. ${ }^{b}$ Instituto de Química. Universidad Católica de Valparaiso. Valparaíso. ${ }^{c}$ Facultad de Farmacia Universidad de Concepción. Concepción - Chile. ${ }^{d}$ Abteilung für Biopharmazie und Pharmazeutische Technologie, Universität des Saarlandes. Saarbrücken - Germany.
\end{abstract}

(Received: December 2, 2009 - Accepted: July 22, 2010)

\begin{abstract}
Chitosan composite films were obtained from acetic or lactic acid chitosan solutions and additives such as glycerol, oleic acid, linoleic acid, polyoxyethylenesorbitan monolaureate (Tween 20) and polyoxyethylenesorbitan monooleate (Tween 80) in order to see the influence of the additive on the film formation, microstructure and morphology. The composite films obtained were observed by Scanning Electron Microscopy (SEM) and Atomic Force Microscopy (AFM) in order to evaluate the different morphology dependent upon the additives. Both SEM and AFM showed the additive influence on the rugosity and morphology of the film.
\end{abstract}

Keywords: Chitosan, composite film, morphology, rugosity.

\section{INTRODUCTION}

Chitosan, the partially deacetylated form of chitin, contains 2-acetamido2-deoxy-beta-D-glucopyranose and 2-amino-2-deoxy-beta-D-glucopyranose.

Chitosan is soluble in aqueous acid solution and because its nontoxicity and biocompatibility, it has been widely applied for biomaterials, for example, as wound dressing, skin grafting template, haemostatic agent, drug delivery vehicle $^{[3,4]}$.

The structure of chitosan is very useful to the synthetic chemist interested in modification due to the differente reactivities of the amino group at the $\mathrm{C} 2$ position and the primary and secondary hydroxyl groups at $\mathrm{C} 6$ and $\mathrm{C} 3$ positions. Several investigators have reported work where selective N-acylchitosan derivatives were prepared ${ }^{[5]}$. Chitosan is readily processible into fibers and films from aqueous acid solution ${ }^{[6]}$.

The modification of solution processed chitosan films at the surface and more than film physical and biological properties would be useful.

Because of its high spatial resolution, and the ability to image under near physiological conditions, atomic force microscopy (AFM) has been used more and more frequently in biological field. Phase imaging is one of the important functions of AFM, which can distinguish physical properties of sample surface, it would seem to be an ideal method by which to study surface roughness. Phase imaging is one of the important functions of AFM, which can distinguish physical properties of sample surface. Phase image shows the phase difference between driving oscillation of the piezoelectric oscillator and oscillation of the cantilevers as the tip interacts with the sample surface. The Atomic Force Microscopy (AFM) is being used to investigate thin and thick film coatings, composites, glasses, synthetic and biological membranes, metals, polymers, and semiconductors and the Scanning Electron Microscopy (SEM) gives information about the topography, morphology and composition of the studied material. ${ }^{[6-10]}$

In this paper, we obtained different chitosan composite films by casting chitosan dispersions with different additives like glycerol, oleic acid, linoleic acid, polyoxyethylenesorbitan monolaureate (Tween 20) and polyoxyethylenesorbitan monooleate (Tween 80 ), in order to compare their influence on the morphological properties. Besides we obtained films from different initial amounts of solution to prepare different thickness films.

\section{EXPERIMENTAL}

\section{Materials}

Chitosan was purchased from Quitoquimica Ltda.. Its degree of deacetylation was $95 \%$ and its Mv was $160.000 \mathrm{~g} / \mathrm{mol}$. Oleic acid, linoleic acid, tween 20 and tween 80 were purchased from Sigma-Aldrich Chemical Company. Acetic acid came from Fisher Scientific Commercial.

\section{Preparation of the Composite Films}

Chitosan solutions ( 1 and $2 \% \mathrm{w} / \mathrm{v})$ were prepared following the next procedure. Either 1 or $2 \mathrm{~g}$. of chitosan were dissolved in an aqueous $1 \%$ acetic acid solution or $1 \%$ lactic acid solution with overnight stirring. The $\mathrm{pH}$ was adjusted at 5.5 units with $5 \%$ sodium hydroxide solution, the other components of the final composite, listed on table 1, were added under mechanical stirring. Finally 20 or $25 \mathrm{~mL}$. of the composite solution were placed into a plate then evaporated inside an oven at $35^{\circ} \mathrm{C}$ overnight ${ }^{[1]}$.

Table 1. Samples of Chitosan Composite Films.

\begin{tabular}{|c|c|c|c|}
\hline \multicolumn{2}{|c|}{ Components } & Level A & Level B \\
\hline \multicolumn{2}{|c|}{ Chitosan Percentage (CHS) } & 1 & 2 \\
\hline \multicolumn{2}{|c|}{ Solvent (SOL) } & acetic acid (AA) & lactic acid (LA) \\
\hline \multirow{2}{*}{} & Glycerol (GLY) & 0 & 0.3 \\
\cline { 2 - 4 } & Oleic acid (OLE) & 0 & 0.005 \\
\cline { 2 - 4 } & Linoleic acid (LIN) & 0 & 0.005 \\
\cline { 2 - 4 } & Tween 20 (T20) & 0 & 0.010 \\
\cline { 2 - 4 } & Tween 80 (T80) & 0 & 0.010 \\
\hline \multirow{2}{*}{} & & & \\
\hline
\end{tabular}

\section{SEM Measurement}

The microstructure and the thickness of the film were imaged using a JSM 6380LV - JEOL Scanning Electron Microscope; front and cross section micrographs were obtained from each sample, the micrographs were taken at $5000 \mathrm{X}$ of magnification for the front side and between 1000X and 3000X of magnification for the cross section. The samples under study were first sputtered with gold during $3 \mathrm{~min}$. to obtain a layer of $150 \mathrm{~A}^{\circ}$ thickness using an Edwards S 150 Sputter Coater.

\section{AFM Measurement}

The topography images of the sample surface were measured with a Multimode Atomic Force Microscope NanoScope IIIa from Vecco Metrology Group, the scanning area was fixed in $5 \mathrm{~mm}$ for all the samples; four images from each sample were obtained in order to calculate the roughness each sample. The measurements were performed in tapping mode using a scanning probe with a force constant of $\sim 40 \mathrm{~N} / \mathrm{m}$ at a resonant frequency of around 170 $\mathrm{kHz}$.

\section{RESULTS AND DISCUSSION}

SEM Analysis.

The front side micrographs are shown in fig. 1 for chitosan acetate film (fig. 1a) and chitosan composite films (fig. 1b-1d).

From fig. 1 it is possible to observe that the highest influence on the morphology of the film is because of the solvent and the presence of the 
additives modifies it but that is a secondary factor; for instance, the presence of tween 20 or tween 80 generates some porosity on the films which is possible to see in fig. 2 with the cross section micrographs of the films, where it is possible to compare the thickness and the microstructure of the films. The size porous ranges from $0.5 \mathrm{um}$ to $1.5 \mathrm{um}$. This is probably due to the presence of the emulsifiers.
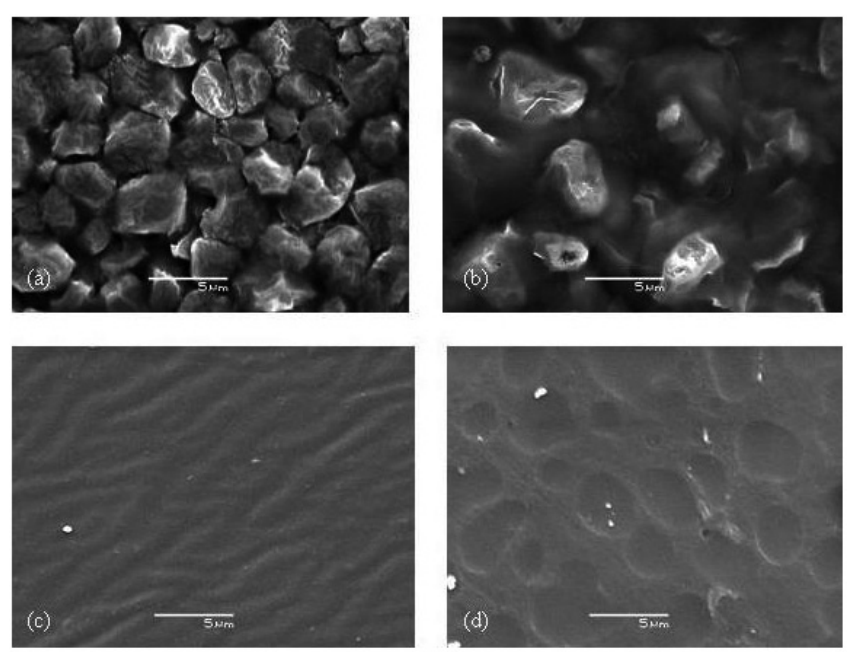

Fig. 1. SEM micrographs of chitosan composite films. (a) chitosan acetate, (b) chitosan acetate with glycerol, (c) chitosan lactate with glycerol, (d) chitosan lactate with glycerol and tween 80 .
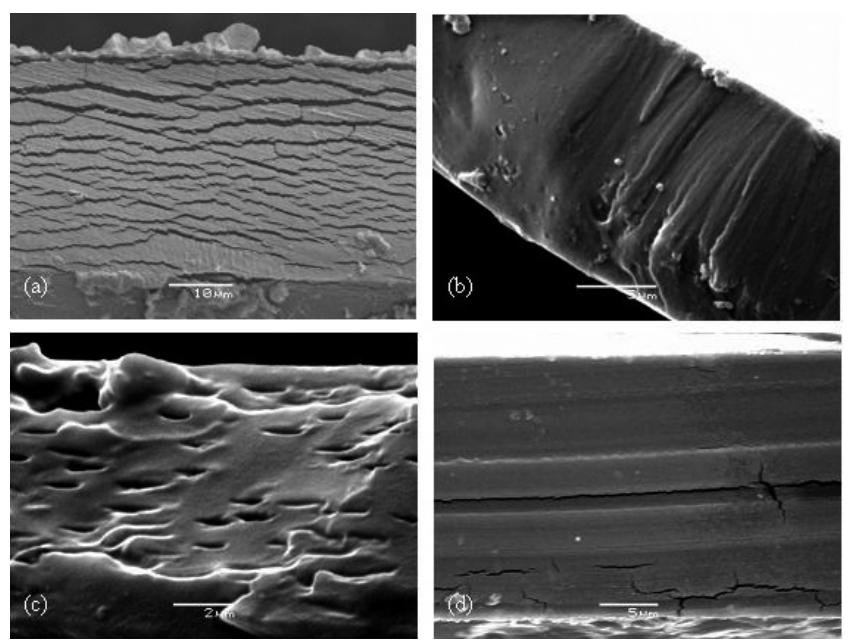

Fig. 2. SEM micrographs of cross section of chitosan composite films. (a) chitosan acetate, (b) chitosan lactate, (c) chitosan lactate with glycerol and tween 20, (d) chitosan acetate with glycerol and oleic acid.

\section{Topography Images of Films.}

Fig. 3 shows the surface plots from the height signal for chitosan acetate film (fig. 3a) and chitosan composite films (fig. $3 b-3$ f); here it is possible to compare the films obtained from chitosan acetate which have less uniform topography so more roughness than those obtained from chitosan lactate; besides, it is possible to observe that the presence of glycerol on the film makes a more homogeneous surface than the film without glycerol due to their plasticizer effect.

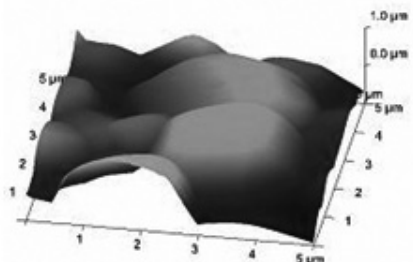

(a)

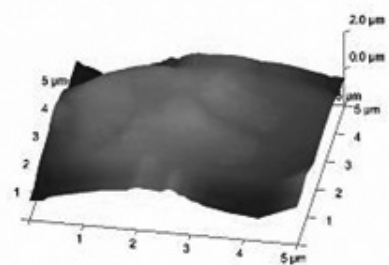

(c)

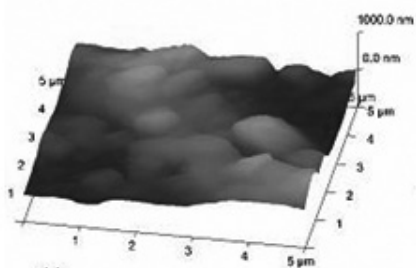

(e)

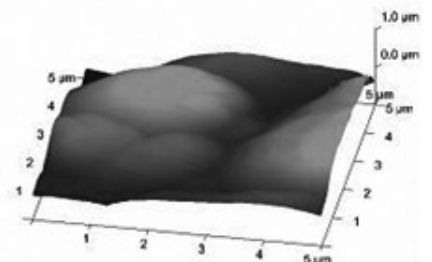

(b)

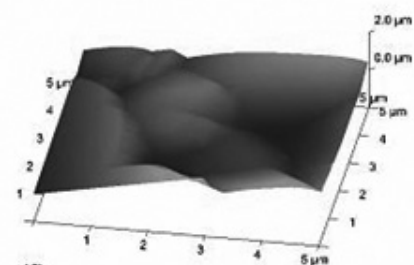

(d)

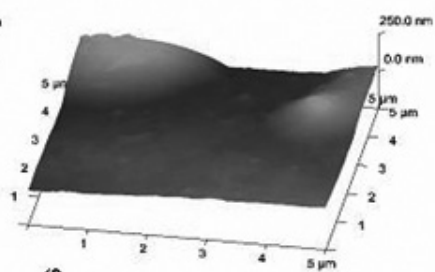

Fig. 3. AFM images of chitosan composite films. (a) chitosan acetate, (b) chitosan acetate with glycerol, (c) chitosan acetate with glycerol and oleic acid, (d) chitosan acetate with glycerol, oleic and linoleic acid, (e) chitosan lactate, (f) chitosan lactate with glycerol and tween 20 .

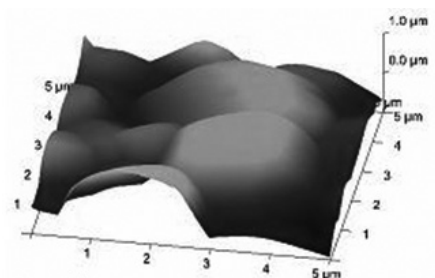

(a)

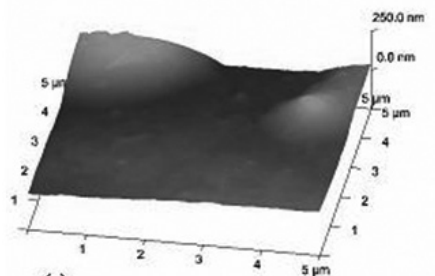

(c)

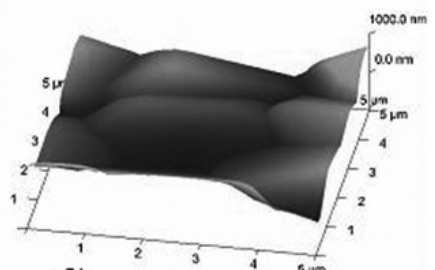

(b)
Fig. 4. AFM images of chitosan composite films. (a) chitosan acetate $1 \%$, (b) chitosan acetate $2 \%$, (c) chitosan lactate with glycerol and tween 20 from $20 \mathrm{~mL}$ of solution, (d) chitosan lactate with glycerol and tween 20 from $25 \mathrm{~mL}$ of solution.

Fig. 4 shows the comparison of the film surface based on the initial volume and chitosan percentage in the solution; from fig. $4 \mathrm{a}$ and $4 \mathrm{~b}$ it is possible to observe that the major percentage of the chitosan in the solution makes the film more uniform. On the other hand, when the volume is increased it is possible 
to see that the film obtained from $25 \mathrm{~mL}$. of composite (5011B) seems to be less uniform than the one obtained from $20 \mathrm{~mL}$. $\left(5011^{\mathrm{a}}\right)$. it could be explained because a major volume of solvent it is necessary major time of drying and the film formed will be more homogenous.

\section{Roughness of the Composites.}

The roughness of each sample was calculated from the images with the NanoScope software, the RMS value is defined as $\sqrt{\sum\left(\mathrm{z}_{\mathrm{i}}-\overline{\mathrm{z}}\right)^{2}}$ and therefore

a measure of the deviation from the mean value of each type of film is showed in table 3. From the values obtained for the roughness it is possible to observe that presence of any additive on the film modifies the roughness but the solvent has the highest influence, for instance from sample 5001 (chitosan acetate) and 5008 (chitosan lactate) it is observed that the chitosan acetate is 10 times less homogeneous than the chitosan lactate, due to their lower porosity and the higher symmetry of lactate.

Table 3. Rugossity of Chitosan Composite Films.

\begin{tabular}{|c|c|c|c|}
\hline Sample & $\mathrm{Ra}(\mathrm{nm})$ & Sample & $\mathrm{Ra}(\mathrm{nm})$ \\
\hline 5001 & 747,7 & $5011 \mathrm{~A}$ & 21,0 \\
\hline 5002 & 405,3 & $5011 \mathrm{~B}$ & 38,4 \\
\hline 5003 & 406,3 & 5012 & 64,4 \\
\hline 5004 & 291,0 & 5013 & 69,0 \\
\hline 5005 & 438,3 & 5014 & - \\
\hline 5006 & 276,9 & 5015 & 218,0 \\
\hline 5007 & 358,7 & 5016 & - \\
\hline 5008 & 87,4 & 5017 & 352,7 \\
\hline 5009 & 52,1 & 5018 & 384,3 \\
\hline 5010 & 53,8 & & \\
\hline & & & \\
\hline
\end{tabular}

In the case of the presence of tween 20 or tween 80 it is possible to observe that the films obtained with tween 20 exhibit less roughness than those obtained with tween 80 , most probably due to the content of oleic acid (70\% approximately) in tween 80 films which does not exist in tween $20(50 \%$ approximately of lauric acid).

The highest roughness was showed for the chitosan acetate film (5001) without any additive from this result, it is possible to confirm that the presence of plasticizers or any additive gives better characteristics like smoothness to the film.

\section{CONCLUSIONS}

The SEM micrographs show that the morphology of the film is mainly related to the solvent, and on the second position to composition and the additives and it is possible to observe that the chitosan lactate films are more homogeneous that the chitosan acetate films.

The AFM images and the roughness value confirm that the chitosan lactate films present less roughness than the chitosan acetate ones, and the presence of the glycerol or any additive on the film makes it more homogeneous than that obtained without any additive and the same solvent.

\section{ACKNOWLEDGEMENTS}

Authors would like to thank the financial support from INNOVA BIO BIO Project 03-B1-212-L1 and UDEC -PUCV 205.024.092.1S project and P.A. thanks CIPA and CONICYT Scholarship.

\section{BIBLIOGRAPHY}

1. G. Cárdenas, P. Anaya, C.von Plessing, C.Rojas and J.Sepulveda, $J$ Mater Sci: Mater Med 19, 2397-2405 (2008).

2. G.Cárdenas, P. Miranda, J. Chil. Chem. Soc. 49:4, 291 - 295 (2004).

3. H.Yang, S. Zhou and X.Deng, Journal of Applied Polymer Science 92, 1625 (2004)

4. M. Huang, L. Liu, G. Zhang, G. Yuan and Y. Fang, International Journal of Biological Macromolecules 38, 191 (2006).

5. K. Kurita, Y. Koyama, S. Chikoka, Polymer J. 20, 1083, 1988.

6. T. D. Rathke, S. M. Hudson, J.Macromol.Sci. Rev. Macromol. Chem. Phys. C34 (3)1994, 375

7. I. Kim, S. Seo, H. Moon, M. Yoo, I. Park, B. Kim and C. Cho, Biotechnology Advances 26, 1-21 (2008).

8. Z. Chen, X. Guo, J. Huang, Y. Hong and Q. Zhang, Anaerobe, 12, $106-$ 109 (2006).

9. S. Alexander, L. Hellemans, O. Marti, J. Schneir, V. Elings and P. Hansma, Journal of Applied Physics, 65:1, 164 (1989).

10. H. Yang, Y. Wang, S. Lai, H.An, Y. Li and F. Chen, Journal of Food Science, 72:4 65-75 (2007)

11. SN. Magonov, J. Cleveland, V.Elings, D. Denley and MH. Wangbo, Surface Science, 389, 201 - 211 (1997).

12. C. Clasen, T. Wilhelms and WM. Kulicke, Biomacromolecules 7:11, 3210 $-3222(2006)$ 